\title{
ADJOINT GROUPS, REGULAR UNIPOTENT ELEMENTS AND DISCRETE SERIES CHARACTERS
}

BY

\author{
G. I. LEHRER
}

\begin{abstract}
It is shown that if $G$ is a finite Chevalley group or twisted type over a field of characteristic $p$ and $U$ is a maximal $p$-subgroup of $G$ then any nonlinear irreducible character of $U$ vanishes on regular elements. For groups of adjoint type the linear content of the restriction to $U$ of a discrete series character $J$ of $G$ is calculated and it is deduced that $J$ takes the value 0 or $(-1)^{S}$ on regular elements of $U(s=\operatorname{rank} G)$.
\end{abstract}

Introduction. This work is concerned primarily with the complex representation theory of finite algebraic groups, although the methods of proof utilise results from algebraic group theory over algebraically closed fields. Two main results are proved: the first is that under appropriate conditions, all nonlinear irreducible characters of the finite unipotent group $U$ vanish on regular unipotent elements (for the definition see next section). This generalizes the result proved in [7] for the unitriangular group. The second result is that if $G$ is one of the finite groups under consideration (e.g. a finite adjoint Chevalley group or twisted type) and $J$ is an irreducible discrete series character of $G$, then the restriction of $J$ to $U$ (a maximal unipotent subgroup) has linear content which is zero or consists precisely of the sum of all the regular linear characters of $U$, each occurring with multiplicity one. The notion of a regular linear character of $U$ was first introduced by Gel'fand and Graev [5] and was translated by the words "general aspect".

These two results hold in particular for the classical linear groups and twisted types and enable one to deduce that the value of an irreducible discrete series character $J$ of such a group on a regular unipotent element is 0 or \pm 1 . Also if $G$ is a linear group over $G F(q)$ then the degree of $J$ is 0 or $\pm 1 \bmod p$, where $q=p^{a}$.

The point of view adopted is to realise $G$ as the group of fixed points of an endomorphism $\sigma$ of the semisimple adjoint group G. The first result (concerning the vanishing of nonlinear characters on regular unipotent elements) is idependent of which group in the isogeny class of $\mathbf{G}$ is chosen, since the finite unipotent subgroups are the same in each case. However the second result (concerning the value of $J$ ) depends essentially on $\mathbf{G}$ being adjoint, as it is necessary to examine the 
action of a split torus $T$ on the set of regular linear characters of $U$. Nevertheless the corresponding value of $J$ on regular unipotent elements for other groups in the isogeny class of $\mathbf{G}$ can be calculated from those given here by the method of [7]. They turn out to be generalized Gaussian sums.

The exposition will deal for the most part with the untwisted case, while the twisted groups are discussed in $\$ 5$.

1. Notation. Let $K$ be an algebraically closed field of characteristic $p>0$ and let $\mathbf{G}$ be a connected semisimple linear algebraic group over $K$ which is defined over the prime field of $K$. Let $\sigma: \mathbf{G} \rightarrow \mathbf{G}$ be an algebraic endomorphism with a finite group $G=\mathbf{G}_{\sigma}$ of fixed points. For any subgroup $\mathbf{H}$ of $\mathbf{G}$ such that $\sigma(\mathbf{H}) \subset \mathbf{H}$ denote by $\mathbf{H}_{\sigma}$ (or $H$ ) its group of fixed points under $\sigma$.

$\mathbf{G}$ contains [9] a Borel subgroup $\mathbf{B}$ and a maximal torus $\mathbf{T} \subset \mathbf{B}$ which are both $\sigma$-stable. Let $\mathbf{U}$ be the unipotent radical of $\mathbf{B}$; then $\mathbf{B}$ is the semidirect product $\mathbf{B}=\mathbf{T} \mathbf{U}$. Denote by $\Sigma$ the root system of $\mathbf{G}$ with respect to $\mathbf{T}$ (assumed irreducible) and let $\Phi$ be the set of fundamental roots of $\Sigma$ in the ordering defined by $\mathbf{B}$. Then for each positive root $\alpha \in \Sigma$ let $\mathbf{X}_{\alpha}$ be the corresponding root subgroup of $U$. It is shown in [1, Theorem 9.8] that there is an isomorphism $x_{\alpha}$ : $K^{+} \rightarrow \mathbf{X}_{\alpha}$ which is rational over the prime field of $K$. $T$ normalizes $\mathbf{X}_{\alpha}$ and for $t \in \mathrm{T}, \zeta \in K$ we have $t x_{\alpha}(\zeta) t^{-1}=x_{\alpha}(\alpha(t) \zeta)$. We suppose throughout that $\mathbf{G}$ is an adjoint group i.e. that the roots $\alpha \in \Sigma$ generate the whole group of rational $K$-characters of $\mathbf{T}$.

If $k$ is the subfield $G F(q)$ of $q$ elements of $K$, then the group of $k$-rational points of $\mathbf{G}$ is realised as $\mathbf{G}_{\sigma}$, where $\sigma$ is the Frobenius endomorphism, given by taking matrix elements to their $q$ th power. In $\S \S 1-4$ we consider such $\sigma$, while in $\S 5, \sigma$ will be more general.

General references for the above material are [2], [4] and [9].

2. Nonlinear characters of unipotent groups. This section is devoted to a proof of the first result mentioned in the introduction, namely

THEOREM A. Let $G$ be a Chevalley group over a finite field $k$ of $q$ elements, and suppose that the characteristic $p$ of $k$ is good for $G$. Then if $U$ is a maximal unipotent subgroup of $G$ and $\rho$ is an irreducible complex character of $U$ of degree greater than one, we have that $\rho(x)=0$ for $x$ any regular unipotent element.

The notation will be as in the section above, and we shall think of $G$ as the set of $k$-rational points of $\mathbf{G}$. The conclusion of Theorem A stands when we replace $G$ by a twisted type (e.g. a finite unitary group $U\left(n, q^{2}\right)$ ) but then for the given proof to apply, it is expedient to assume that $\mathbf{G}$ is simple, i.e. that the root system $\Sigma$ is irreducible. For simplicity, the argument is given here for the untwisted case and in the last section we indicate how to modify the proof for the 
twisted groups. We shall moreover assume that $\Sigma$ is irreducible without loss of generality.

Bad primes are discussed in $[9$, p. 178]. The result is false for these, but this is not a great restriction. The bad primes are those which divide the coefficients of the fundamental roots in the expression of the highest root as a linear combination of fundamental roots. For the various irreducible root systems they are as follows:

(i) Type $A_{r}$ : none;

(ii) Types $B_{r}, C_{r}, D_{r}: 2$;

(iii) Types $E_{6}, E_{7}, F_{4}, G_{2}: 2$ and 3;

(iv) Type $E_{8}: 2,3$ and 5 .

The prototype of a regular unipotent element is a unitriangular matrix whose Jordan form consists of a single block. In general an element $\boldsymbol{x}$ of $\mathbf{G}$ is regular if its centraliser in $\mathbf{G}$ has dimension $r$ equal to the rank of $\mathbf{G}$. Such elements were studied comprehensively by Steinberg in [11] where he proved the existence of (among other things) regular unipotent elements in the subgroup $G$ of $\mathbf{G}$. For convenience we collect together results necessary for the proof of Theorem $A$ as a sequence of lemmas. Recall that $\Sigma$ is the irreducible root system of $\mathbf{G}$ with respect to $T$ and $\Phi=\left\{\alpha_{1}, \ldots, \alpha_{r}\right\}$ is the set of fundamental roots corresponding to the ordering mentioned in the previous section. We denote by $\Sigma^{+}$the set of positive roots with respect to this ordering.

LEMMA 2.1. (i) With notation as in the previous section, let $x=$ $\Pi_{\alpha \in \Sigma}+x_{\alpha}\left(\xi_{\alpha}\right)$ be an element of $U$. Then $x$ is regular if and only if $\xi_{\alpha} \neq 0$ for $\alpha \in \Phi$ (i.e. for fundamental roots $\alpha$ ).

(ii) If the $x$ of (i) is regular, it is contained in a unique Borel subgroup (conjugate of $\mathrm{B}=\mathrm{T} \mathrm{U}$ ).

(iii) If the element $x$ in (1) is regular, then the centraliser $\mathbf{G}_{\boldsymbol{x}}$ of $\boldsymbol{x}$ in $\mathbf{G}$ is $\mathbf{U}_{\boldsymbol{x}}$ (i.e. is contained in $\mathbf{U}$ ), provided that $\mathbf{G}$ is adjoint.

Proof. These results are essentially due to Steinberg [11, 3.1, 3.2 and 3.3] although (iii) is explicitly proved by Springer in $[8,4.3]$. For the reader's convenience (and future reference) we sketch a proof of (ii) and (iii), assuming (i). The proof depends on the Bruhat decomposition $G=\bigcup B n_{w} B$ [4, Expose 13] of $G$ as a disjoint union of $(B, B)$ double cosets, where the $n_{w}$ are representatives of $W$ in the normalizer of $\mathbf{T}$ (see $\S 1$ ), and on the Chevalley commutator formula.

If for some $g \in \mathbf{G}, x \in g^{-1} \mathbf{B} g$ then $g=b n_{w} b^{\prime}\left(b, b^{\prime} \in \mathbf{B}, w \in W\right)$ and we may take $g=n_{w} b^{\prime}$ since $b^{-1} \mathrm{~B} b=\mathrm{B}$. But then $g x g^{-1} \in \mathrm{B}$ and since $b^{\prime} x b^{1-1}$ is a regular element of type (i) (by the commutator formula) we may take $g=n_{w}$. Now $g x g^{-1}=\Pi_{\alpha \in \Sigma}+x_{w(\alpha)}\left(\xi_{\alpha}^{\prime}\right)$ since $n_{w} X_{\alpha} n_{w}^{-1}=X_{w(\alpha)}$ and $\xi_{\alpha}^{\prime} \neq 0$ if $\xi_{\alpha} \neq 0$. 
Hence for each fundamental $\alpha, \xi_{\alpha} \neq 0$ implies that $w(\alpha)$ is a positive root (here one uses the fact that $\mathbf{U}=\mathbf{U}_{w}^{-} \mathbf{U}_{w}^{+}$, a consequence of the commutator formula). Thus $g \in \mathbf{B}$ and (ii) is proved.

The above argument also shows that the centraliser $\mathbf{G}_{\boldsymbol{x}}$ of $x$ in $\mathbf{G}$ is contained in B. Further if $g \in \mathbf{B} \cap \mathbf{G}_{x}$ and $g=u t \quad(u \in \mathrm{U}, t \in \mathrm{T})$ then $\mathrm{gxg}^{-1}=$ $\Pi_{\alpha} x_{\alpha}\left(\xi_{\alpha}^{\prime}\right)$ and for $\alpha$ fundamental, $\xi_{\alpha}^{\prime}=\alpha(t) \xi_{\alpha}$. This follows from the commutator formula, which shows that $u$ does not change $\xi_{\alpha}$ for $\alpha$ fundamental, and from the formula $t x_{\alpha}(\xi) t^{-1}=x_{\alpha}(\alpha(t) \cdot \xi$ ) (see $\S 1$ ). Thus we have $\alpha(t)=1$ for $\alpha \in$ $\Phi$. But in an adjoint group the fundamental roots form a Z-linear basis of the character group $X(\mathrm{~T})$. Hence $t=1$ and $g=u \in \mathrm{U}_{x}$.

Lemma 2.2. Let $G$ be the set of $k$-rational points of $\mathbf{G}$. Then $G$ contains $a$ regular unipotent element $x$ of $\mathbf{G}$ and $x$ lies in a unique (G.) conjugate of $B$ (the group of rational points of $\mathbf{B})$.

Proof. By Lemma 2.1(i) the element $x=x_{\alpha_{1}}(1) x_{\alpha_{2}}(1) \ldots x_{\alpha_{r}}(1)$ is regular unipotent $\left(\Phi=\left\{\alpha_{1}, \ldots, \alpha_{r}\right\}\right)$ and since the $x_{\alpha}$ are assumed to be $k$-rational morphisms [1, Theorem 9.8], $x \in G$. Now $x$ lies in a unique conjugate of $B$, so that it remains to show that if $x \in g B g^{-1}$ then $g \in B$. But $x \in g B g^{-1}$ implies that $g \in \mathbf{B}$ by the argument in the proof of Lemma 2.1. Hence $g \in \mathbf{B} \cap G=B$.

LEMMA 2.3. The number of regular unipotent elements in $G$ is $|G| / q^{r}$ where $q=|k|$ and $r=\operatorname{rank}(\mathbf{G})$.

Proof. This result appears (more generally) in $[9$, p. 222]. Since each regular unipotent element is in a unique conjugate of $B$, the total number of them is $n_{1} n_{2}$ where $n_{1}=$ the number of conjugates of $B$ in $G$ and $n_{2}=$ the number of regular unipotent elements in $B$. Now $B$ is self-normalizing by the proof of Lemma 2.2 (since $g$ normalizes $B$ implies that $g x g^{-1} \in B$ ) and so $n_{1}=|G| /|B|$. To find $n_{2}$, we use Lemma 2.1(i) and the fact that $x_{\alpha}$ are $k$-rational. The latter implies that the unipotent elements of $B$ are precisely the elements $x=\Pi_{\alpha \in \Sigma}+x_{\alpha}\left(\xi_{\alpha}\right)$ with $\xi_{\alpha} \in k$, and the former says that $x$ is regular if and only if $\xi_{\alpha} \neq 0$ for $\alpha \in$ $\Phi$. Hence

$$
n_{2}=|U|(q-1)^{r} / q^{r}=|U||T| / q^{r}
$$

since $|T|=(q-1)^{r}$ (T is $k$-split). The result follows.

LEMMA 2.4. All the regular unipotent elements of $G$ are conjugate in $G$.

This result is due to Springer [8, Theorem 4.14]. It depends essentially on the facts that $\mathbf{G}$ is adjoint and that the characteristic $p$ of $k$ is good. These imply that $\mathbf{G}_{\boldsymbol{x}}$ is connected which gives the result by a general argument. If $\mathbf{G}$ were not adjoint $\mathbf{G}_{\boldsymbol{x}}$ would have a finite direct factor equal to the centre of $\mathbf{G}$, while if $\boldsymbol{p}$ were bad, $\mathbf{U}_{\boldsymbol{x}}$ may be disconnected. 
PROPOSITION 2.5. Let $G$ be the group of $k$-rational points of the connected semisimple adjoint group $\mathbf{G}$ and suppose that the characteristic of $k$ is good for $\mathbf{G}$. Then if $x$ is a regular unipotent element of $\mathbf{G}$ contained in $U$, we have $\left|U_{x}\right|=q^{r}$.

Proof. By Lemma 2.4 all the regular unipotent elements of $G$ are conjugate in $G$, and hence their number is $|G| /\left|G_{x}\right|$, where $G_{x}$ is the centraliser in $G$ of the fixed regular unipotent element $x$. But Lemma 2.1(iii) shows that $G_{x}=G \cap$ $\mathbf{G}_{x}=G \cap \mathbf{U}_{x}=U_{x}$. Hence by Lemma 2.3 we have $|G| /\left|U_{x}\right|=|G| / q^{r}$, from which we see $\left|U_{x}\right|=q^{r}$.

We now turn to the linear characters of $U$. The number of distinct linear (i.e. one-dimensional) complex characters of $U$ is $|U| /\left|U^{\prime}\right|$ where $U^{\prime}$ is the commutator subgroup of $U$. To determine this number we have

LEMma 2.6. Suppose that the characteristic of $k$ is good for $\mathbf{G}$. Then

(i) $U^{\prime}$ is the product of the groups $X_{\alpha}$ of rational points of the root subgroups $\mathbf{X}_{\alpha}$ with $\alpha$ positive and not fundamental.

(ii) The number of distinct linear complex characters of $U$ is $q^{r}$.

PRoof. (i) This is part of the content of [6, Lemma 7]. Note that 2 is a bad prime for types $B_{r}, C_{r}, F_{4}$ and $G_{2}$, and 3 is bad for $G_{2}$.

(ii) From (i), $U / U^{\prime}$ is generated by the $X_{\alpha}$ with $\alpha \in \Phi$, and they commute modulo $U^{\prime}$. Hence

$$
\left|U / U^{\prime}\right|=\prod_{\alpha \in \Phi}\left|X_{\alpha}\right|=q^{r} .
$$

Proof of Theorem A. The statement of the theorem depends only on $U$, and so is not affected if we replace $G$ by a group "isogenous" to it. Hence we may take $G$ as in the statement of Proposition 2.5. Now for any finite group $G$ and $x \in G$ we have

$$
\sum_{x}|\chi(x)|^{2}=\left|G_{x}\right|
$$

where the sum is over the irreducible complex characters $\chi$ of $G$ and $G_{x}$ is the centraliser of $x$ in $G$. This is from the dual Schur orthogonality relations for complex characters. Applying this formula to the regular unipotent element $x$ of the group $U$, we see that since for each linear character $\chi$ of $U$ we have $|\chi(x)|=1$ $(\chi(x)$ is a root of unity), the contribution of the linear characters to the left-hand side above is $q^{r}$ by Lemma 2.6. But $\left|U_{x}\right|=q^{r}$ by Proposition 2.5 and so we have $\Sigma_{\operatorname{deg} \rho>1}|\rho(x)|^{2}=0$ where the sum is over the irreducible nonlinear complex characters of $U$. Thus $\rho(x)=0$ for each such character. Q.E.D.

3. Regular linear characters. It is apparent from Lemma 2.6 that the group $U / U^{\prime}$ (where $U$ is a maximal $p$-subgroup of the Chevalley group $G$ ) is isomorphic to a direct product of $r$ copies of $k^{+}$. A linear complex character of $U$ is therefore given by a sequence $\left(\chi_{1}, \chi_{2}, \ldots, \chi_{r}\right)$ of characters of $k^{+}$, where 


$$
\left(\chi_{1}, \chi_{2}, \ldots, \chi_{r}\right)\left(\prod_{\alpha \in \Sigma^{+}} x_{\alpha}\left(\xi_{\alpha}\right)\right)=\prod_{i=1}^{r} \chi_{i}\left(\xi_{\alpha_{i}}\right),
$$

so that $\chi_{i}$ "acts on" the fundamental root subgroup $X_{\alpha_{i}}$.

Definition. We say the linear character $\left(\chi_{1}, \ldots, \chi_{r}\right)$ of $U$ is regular if none of the $\chi_{i}$ is the identity (trivial) character of $k^{+}$.

Gel'fand and Graev were the first to notice the importance of these in the study of discrete series characters of $G$ [5]. They called such characters "general aspect" (in translation) but it seems more appropriate to refer to them as regular, in analogy with the term for unipotent elements.

Given a nontrivial linear character $\lambda$ of $k^{+}$, we define the translation of $\lambda$ by $a \in k$ as $\lambda^{a}$ where $\lambda^{a}(b)=\lambda(a b)$. It is clear that all $|k|=q$ characters of $k^{+}$ are of the form $\lambda^{a}$ as $a$ ranges over the $q$ elements of $k$ (" $k$ is self-dual"). Thus with reference to the fixed nontrivial element $\lambda$ of the character group of $k^{+}$, the linear characters of $U$ can be identified as sequences $\left(a_{1}, a_{2}, \ldots, a_{r}\right)$ of elements of $k$, where $\chi_{i}=\lambda^{a_{i}}$, and translation by $a \in k$ corresponds to multiplication by $a$. The condition that $\left(a_{1}, \ldots, a_{r}\right)$ represent a regular character is then that $a_{i} \neq 0$ for $i=1,2, \ldots, r$.

The torus $T$ normalizes $U$ and acts according to

$$
t \prod_{\alpha \in \Sigma+} \chi_{\alpha}\left(\xi_{\alpha}\right) t^{-1}=\prod_{\alpha \in \Sigma+} \chi_{\alpha}\left(\alpha(t) \cdot \xi_{\alpha}\right) . \quad(t \in T) .
$$

Further $T$ acts on the set of linear characters of $U$ according to

$$
\left(\chi_{1}, \ldots, \chi_{r}\right)^{t}(u)=\left(\chi_{1}, \ldots, \chi_{r}\right)\left(t u t^{-1}\right)
$$

where $u \in U, t \in T$.

LEMMA 3.4. With the notation introduced above, we have

$$
\left(a_{1}, a_{2}, \ldots, a_{r}\right)^{t}=\left(\alpha_{1}(t) a_{1}, \alpha_{2}(t) a_{2}, \ldots, \alpha_{r}(t) a_{r}\right)
$$

where $a_{i} \in k$ and $t \in T$.

(ii) $T$ sends regular characters to regular characters.

PROof. (i) is a restatement of (3.2) and (3.3) in the notation above. (ii) follows because $\alpha_{i}(t) \neq 0$ for $i=1,2, \ldots, r$.

THEOREM B. Suppose $\mathbf{G}$ is as in $\$ 1$ (i.e. adjoint). Then $T$ permutes the regular linear characters of $U$ transitively.

PRoof. We show that every character is a $T$-transform of $(1,1, \ldots, 1)$ (which corresponds to $(\lambda, \lambda, \ldots, \lambda)$ ). From Lemma 3.4 we see that if $t \in T$ then $(1,1, \ldots, 1)$ is mapped by $t$ to the character $\left(\alpha_{1}(t), \alpha_{2}(t), \ldots, \alpha_{1}(t)\right)$. Consider the action of $T$ on the orbit of $(1,1, \ldots, 1)$. If $t \in T$ fixes $(1,1, \ldots$, 1) then we have $\alpha_{i}(t)=t(i=1,2, \ldots, r)$. Hence $t \in \operatorname{ker} \alpha_{i}(i=1,2, \ldots, r)$, where $\alpha_{i}$ is regarded as a character: $\mathrm{T} \rightarrow K$. But since $\mathbf{G}$ is adjoint, the $\alpha_{i}$ gener- 
ate the character group $X(\mathrm{~T})$ and hence $t$ is annihilated by each character of $\mathrm{T}$. Thus $t=1$.

The number of regular characters in the orbit of $(1,1, \ldots, 1)$ is therefore $|T|=(q-1)^{r}$. But this is the total number of distinct regular characters, whence it follows that each one is in the $T$-orbit of $(1,1, \ldots, 1)$. Q.E.D.

4. Discrete series characters. Among the irreducible complex characters of $G$ is a family of distinguished characters, called discrete series, from which in principle it is possible to find all characters by solving ramification problems, i.e. decomposing induced characters. In this section we show that on the regular unipotent element $x$, these take the value 0 or \pm 1 and that their degree modulo $q$ is also 0 or \pm 1 . In order to define irreducible discrete series characters we recall some facts about parabolic subgroups of $G$. For details the reader is referred to [2] and [4].

A standard parabolic subgroup of $\mathbf{G}$ corresponds to a subset $S$ of the fundamental roots. If $W_{S}$ is the subgroup of the Weyl group $W$ generated by reflections corresponding to $S$, the corresponding parabolic subgroup is $\mathbf{G}_{S}=\mathbf{B} W_{S} \mathbf{B}$. Now $\mathbf{G}_{S}$ has a maximal normal unipotent subgroup $\mathbf{U}_{S}$, called the unipotent radical of $\mathbf{G}_{S}$, and is a semidirect product $\mathbf{G}_{S}=\mathbf{M}_{S} \mathbf{U}_{S}$, where $\mathbf{M}_{S}$ is called a Levi radical of $\mathbf{G}_{S}$ and the product is called the Levi decomposition. One can make similar statements when the groups are replaced by their groups of $k$-rational points.

Definition. The irreducible complex character $J$ of $G$ is discrete series if $J$ is not a component of the induced character $1_{U_{S}}^{G}$ for any proper subset $S$ of $\Phi$, where $U_{S}$ is the group of rational points of $U_{S}$. (We refer to $U_{S}$ also as the unipotent radical of $G_{S}$.)

The groups $U_{S}$ are products of root subgroups $X_{\alpha}$. To describe which roots $\alpha$ occur we introduce the torus $\mathrm{T}_{S}=\bigcap_{\alpha \in S} \operatorname{ker} \alpha$ for any subset $S$ of $\Phi$ (the set of fundamental roots). Then we have

LEMMA 4.1. $U_{S}$ is the product of the root subgroups $X_{\beta}$ where $\beta$ is positive and the restriction $\left.\beta\right|_{\mathrm{T}_{S}}$ is nontrivial.

Proof. For a complete proof the reader is referred to [2]. The reason why one should expect this result is that $M_{S}$ contains that part of $U$ which is generated by the $X_{\beta}$ with $\beta$ trivial on $\mathrm{T}_{S}$ and $U_{S}$ is its complement in $U$.

Corollary $4.1^{\prime}$. $U_{S}$ is the product of the root subgroups $X_{\beta}$ where $\beta$ is positive and contains fundamental roots in $\Phi-S$ in its expression as a linear combination of fundamental roots (see [3, p. 118]).

Proposition 4.2. Let $J$ be an irreducible discrete series character of $G$. If the linear character $\chi$ of $U$ is a component of the restriction $\left.J\right|_{U}$, then $\chi$ is a regular linear character of $U$. 
Proof. Suppse $\left.J\right|_{U}$ contains the linear character $\chi=\left(\chi_{1}, \chi_{2}, \ldots, \chi_{r}\right)$ (in the notation of $\S 3$ ) and that $\chi$ is not regular, so that for some $i, \chi_{i}$ is trivial. Then $\left.\chi\right|_{X_{\alpha_{i}}}$ is the identity character of $X_{\alpha_{i}}$, where $\alpha_{i}$ is the fundamental root corresponding to the $i$-component of $\chi$. Let $U_{i}$ be the subgroup of $U$ generated by the root subgroups $X_{\beta}$ where the expression for $\beta$ as a linear combination of the fundamental roots contains $\alpha_{i}$ with nonzero coefficient. By the commutator formula $U_{i}$ is a normal subgroup of $U$ and $X_{\alpha_{i}}$ is the unique fundamental root subgroup in $U_{i}$. Thus $\left.\chi\right|_{U_{i}}$ is the identity character of $U_{i}$ since $\chi$ acts as identity on root subgroups $X_{\beta}$ with height $\beta>1$. But, by Corollary $4.1^{\prime}, U_{i}$ is precisely the unipotent radical $U_{S}$ where $S=\Phi-\left\{\alpha_{i}\right\}$ and so the restriction $\left.J\right|_{U_{S}}$ contains $\left.\chi\right|_{U_{S}}$ which is the identity character. By Frobenius reciprocity, we see that $J$ is a component of the induced character $1_{U_{S}}^{G}$, which contradicts the discrete series nature of $J$. Hence if $\chi$ is a linear component of $\left.J\right|_{U}$ then $\chi$ is regular. Q.E.D.

LEMMA 4.3. Let $\chi$ be a regular linear character of $U$. Then the induced character $\chi^{G}$ is multiplicity free (i.e. all the irreducible components occur with multiplicity 1),

This is a theorem due to Gel'fand and Graev who stated it and proved it for $G=S L(n, q)$ in [5]. A general proof which includes the twisted groups is given by Steinberg in [12, Theorem 49].

THEOREM C. Let $J$ be an irreducible discrete series character of $G$, where $\mathbf{G}$ is adjoint. Then we have a dichotomy: either

(a) the restriction of $J$ to $U$ contains no linear character of $U$, or

(b) the restriction of $J$ to $U$ contains each regular linear character of $U$ with multiplicity one, and contains no other linear characters of $U$.

Proof. Suppose $\left.J\right|_{U}$ contains a linear character $\chi$ of $U$. Then by Proposition 4.2, $\chi$ is regular. By Frobenius reciprocity, $J$ is a component of $\chi^{G}$ and Lemma 4.3 shows that $J$ occurs with multiplicity one in $\chi^{G}$, from which it follows that $\chi$ has multiplicity one in $\left.J\right|_{U}$.

Now $J$ is a class function on $G$ and so in particular $J$ is invariant under conjugation by $T$. Thus $\left.J\right|_{U}$ is invariant under conjugation by $T$ (recall that $T$ normalizes $U$ ) and hence the irreducible constituents of $\left.J\right|_{U}$ are permuted by $T$. But from Theorem B, each regular linear character of $U$ is conjugate to $\chi$ under $T$ whence $\left.J\right|_{U}$ contains each regular character of $U$. Moreover the argument given for $\chi$ shows that the multiplicity of a regular character is one, and no other linear characters of $U$ occur. Thus situation (b) pertains. Q.E.D.

From Theorem $\mathrm{C}$ we deduce easily the value of $J$ on a regular unipotent element: 
THEOREM D. Suppose that $J$ is an irreducible discrete series character of $G$ and that $x$ is a regular unipotent element of $G$. Then in case (a) above, $J(x)=0$ while in case (b), $J(x)=(-1)^{r}$, where $r=\operatorname{rank}(G)$. Here we assume that the characteristic of $k$ is good for $G$.

Proof. Since all regular unipotent elements of $G$ are conjugate in $G$ (Lemma 2.4) we may take $x$ in $U$, and calculate $J(x)$ by observing that $J(x)=\left.J\right|_{U}(x)$. By Theorem A, if $\rho$ is a nonlinear irreducible character of $U, \rho(x)=0$; hence to calculate $J(x)$ we require only the linear content of $\left.J\right|_{U}$ which is furnished by Theorem C. If $\left.J\right|_{U}$ contains no linear characters (case (a)) then $J(x)=0$. If $\left.J\right|_{U}$ contains each of the $(q-1)^{r}$ regular linear characters of $U$ (case (b)) then (taking $x=\prod_{i=1}^{r} x_{\alpha_{i}}(1)$ without loss since all regular unipotent $x$ are conjugate) we have

$$
J(x)=\sum_{\left(a_{1}, \ldots, a_{r}\right)} \lambda^{a_{1}} \ldots \lambda^{a_{r}}(x)=\sum_{\left(a_{1}, \ldots, a_{r}\right)} \lambda\left(a_{1}\right) \ldots \lambda\left(a_{r}\right)
$$

where $\lambda$ is the fixed nontrivial character of $k^{+}$referred to in $\S 3$ and the sum is over sequences $\left(a_{1}, \ldots, a_{r}\right)$ with $a_{i} \in k^{*}$. But $\Sigma_{a \neq 0} \lambda(a)=-1$, whence it follows that $J(x)=(-1)^{r}$ as required.

It is noteworthy that both cases (a) and (b) do occur, the case (b) being apparently more common. All discrete series characters of $\operatorname{PGL}(n, q)$, for example, are type (b), while $\operatorname{Sp}(4, q)$ has a discrete series character of type (a). The latter is a counterexample (of which Kneser's original is a special case) to Gel'fand and Graev's now famous erroneous statement that each irreducible character of $G$ contains a linear character of $U$.

Corollary. Let $G$ and $J$ be as in Theorem D. Then the degree of $J \equiv 0$ or $(-1)^{r}$ modulo $p$.

Proof. This follows because the degree of any nonlinear irreducible character of $U$ is divisible by $p$. Hence in case (a) $J(1) \equiv 0 \bmod p$ and in case (b) $J(1) \equiv(q-1)^{r} \equiv(-1)^{r} \bmod p$.

5. The twisted case. In this section we show how the arguments presented earlier are modified to give results for the twisted Chevalley groups (or "Steinberg variations"), e.g. $P G U\left(n, q^{2}\right)$. To realise these groups, we take the same $\mathbf{G}$ as in $\S 1$ (i.e. $\mathbf{G}$ is a connected, simple adjoint group over $K$ ) but instead of the Frobenius endomorphism, we take $\sigma$ to be any endomorphism of $\mathbf{G}$ such that $\sigma$ fixes $\mathbf{T}$ and $\mathbf{U}$ and the group $G=\mathbf{G}_{\sigma}$ of points fixed by $\sigma$ is finite (e.g. to obtain the unitary groups, compose the Frobenius map by taking the inverse transpose of matrices and conjugation by an appropriate matrix).

Then a certain power $q$ of the characteristic $p$ of $K$ arises naturally as follows:

LEMma 5.1. Suppose $\mathbf{G}$ is simple and $\sigma$ is as above. Then there is a power 
$q$ of $p$ and permutation $\theta$ of $\Sigma^{+}$such that $\sigma\left(x_{\alpha}(k)\right)=x_{\alpha}\left(c_{\alpha} k^{q}\right)$ for all $k \in K$ where $c_{\alpha}= \pm 1$ and $c_{\alpha}=+1$ for $\alpha$ fundamental.

Proof. This follows from [10, $\S: 1.2$ and 11.14] and [12, Theorem 29].

One can then show (see $[9$, p. 222]) that the number of regular unipotent elements of $G\left(=\mathbf{G}_{\sigma}\right)$ is $|G| / q^{r}$. Moreover if the characteristic $p$ is good for $\mathbf{G}$, there is a single conjugacy class of regular unipotent elements in $G[9$, p. 221]. These facts furnish a proof of Proposition 2.5 for the twisted case, namely that here also if $x(\in U)$ is a regular unipotent element of $G$ and $U_{x}$ is its centriliser in $U$, then $\left|U_{x}\right|=|G| / q^{r}$.

In the twisted case, the relationship between the root structures of $\mathbf{G}$ and $G$ is not quite so straightforward, but we do have $\left|U / U^{\prime}\right|=q^{r}$; this will transpire from the following discussion of the structure of $U / U^{\prime}$, which is also necessary before making the modifications in the definitions of regular linear characters of $U$ and discrete series characters of $G$ which are appropriate to the twisted case. For the following facts regarding the structure of the twisted groups, see Steinberg $[12, \S 11]$ and $[10, \S 11]$.

The permutation $\theta$ of Lemma 5.1 permutes the fundamental roots, and if $\theta_{1}, \theta_{2}, \ldots, \theta_{s}$ are the orbits of fundamental roots, we denote by $\mathbf{X}_{i}(i=1$, $2, \ldots, s)$ the product in $\mathbf{U} / \mathbf{U}^{\prime}$ of the fundamental root subgroups $\mathbf{X}_{\alpha}$ with $\alpha \in$ $\theta_{i}$. We then have:

LEMMA 5.2. Suppose that $p$ is good for G. Then

(i) $X_{i}$ is fixed by $\sigma$ and if $X_{i}=X_{i \sigma}$ then $\left|X_{i}\right|=q^{n_{i}}$, where $n_{i}$ is the number of fundamental roots in the orbit $\theta_{i}$.

(ii) The group $U / U^{\prime}$ is the direct product of the $X_{i}(i=1,2, \ldots, s)$, and is elementary abelian.

Proof. (i) The argument of [6, Lemma 7] shows that in good characteristic, $\mathbf{U}^{\prime}$ is the product of the root subgroups $\mathbf{X}_{\alpha}$ for $\alpha$ nonfundamental. Moreover $\sigma\left(U^{\prime}\right) \subset U^{\prime}$. The statement (i) now follows from [10, §11.8].

(ii) By $[10, \S 10.11]$ the natura? map: $U \rightarrow\left(U^{\prime} / U^{\prime}\right)_{\sigma} \cong \Pi_{i=1}^{s} X_{i \sigma}$ is surjective. Hence we have an epimorphism $\eta: U / U^{\prime} \rightarrow \Pi_{i=1}^{s} X_{i}$. Now by $[10, \S 11.8]$ we have

$$
\left|U / U^{\prime}\right|=q^{r}=q^{n_{1}+n_{2}+\ldots+n_{s}}=\prod_{i=1}^{s}\left|X_{i}\right| .
$$

Hence $\eta$ is an isomorphism as required.

Putting this together with the twisted version of Proposition 2.5 we have:

THEOREM $\mathrm{A}^{\prime}$. Let $G$ be a finite Chevalley group or Steinberg group twisted from a group $\mathbf{G}$ over $K$ with irreducible root system $\Sigma$. Let $U$ be a maximal $p$ subgroup of $G$ (where $p=$ characteristic of $K$ ). Then if $p$ is good for $\mathbf{G}$ and $\rho$ is 
a nonlinear irreducible complex character $U, \rho(x)=0$ for all regular unipotent elements $x$ of $U$.

One can be more explicit about the groups $X_{i}$, using Lemma 5.1. Let $\left\{\beta_{1}, \ldots, \beta_{t}\right\}$ form the $\theta$-orbit $\theta_{i}$ of fundamental roots where $\theta$ permutes the $\beta_{i}$ cyclically. Then by Lemma $5.1, x_{\beta_{1}}\left(k_{1}\right) x_{\beta_{2}}\left(k_{2}\right) \ldots x_{\beta_{t}}\left(k_{t}\right) \in X_{i}$ if and only if $k_{2}=k_{1}^{q}, k_{3}=k_{2}^{q}, \ldots, k_{t}=k_{t-1}^{q}, k_{1}=k_{t}^{q}=k_{1}^{q t}$. Hence $k_{1} \in G F\left(q^{t}\right)$ and $k_{2}$, $k_{3}, \ldots, k_{t}$ are determined by $k_{1}$.

This shows that an element of $U$ is regular (see Lemma 2.1) if it has a nontrivial projection onto $X_{i}$ for $i=1,2, \ldots, s$. Regular characters are defined similarly.

DEFinition. The linear character $\chi$ of $U$ is regular if it has nontrivial restriction to each $X_{i}(i=1,2, \ldots, s)$.

The torus $T$ acts on $U / U^{\prime}$ and hence on the set of linear characters of $U$, as in $\S 3$, and we see as in the proof of Theorem $B$ that $t \in T$ stabilizes a regular linear character if and only if $t \in \bigcap_{\alpha \in \Phi} \operatorname{ker} \alpha$. Hence if $\mathbf{G}$ is adjoint $t=1$. Moreover by $[8, \S 11.10]$ we have $|T|=\prod_{i=1}^{s}\left(q^{n_{i}}-1\right)$.

Since the number of regular characters is clearly equal to this, we have shown:

THEOREM B'. Let $G$ be a finite Chevalley group or twisted type as in Theorem $\mathrm{A}^{\prime}$. Then $T$ permutes the regular linear characters of $U$ transitively.

The next result, concerning discrete series characters, also remains valid. Here we simply replace the groups $G_{S}, M_{S}$ and $U_{S}$ of rational points by corresponding groups of points fixed by $\sigma$. The proof of Proposition 4.2 must be modified so that instead of root subgroups we have subgroups corresponding to $\theta$-orbits of roots, but the conclusion remains valid. The theorem that if $\chi$ is a regular linear character of $U$ then the induced character is multiplicity free (corresponding to Lemma 4.3) is proved by Steinberg in $[12$, p. 258 et seq.]. Thus we have

THEOREM $C^{\prime}$. Let $J$ be an irreducible discrete series character of $G$, where $G$ is as in Theorem $\mathrm{A}^{\prime}$. Then the conclusion of Theorem $\mathrm{C}$ holds.

The proof of Theorem D remains unchanged, as we have noted that $X_{i} \cong$ $G F\left(q^{n^{i}}\right)^{+}$.

THEOREM $D^{\prime}$. With $G$ and $J$ as above and $x$ a regular unipotent element of $U$ we have either $J(x)=0$ or $J(x)=(-1)^{s}$.

Corollary (i) remains unchanged but in Corollary (ii) it is necessary to remark that the root system of $G$ may be distinct from that of $\mathbf{G}$. It is defined in terms of the orbits $\theta_{i}$ of fundamental roots (see $[10, \S 1]$ ), and it is for this root system that the condition in Corollary (ii) must be stated. 
6. Concluding remarks. If $p$ is a bad prime for $\mathbf{G}$ and $x$ a regular unipotent element of $G$, then the centraliser $U_{x}$ is not connected; in fact $x$ is not in the identity component of $U_{x}([8$, Theorem 4.12]). Hence there is more than one class of regular unipotent elements, and the author knows of no results here. Moreover there may be pathology in the structure of $U / U^{\prime}$ in bad characteristic for the twisted cases and Theorem A fails here. Thus for bad characteristic the results presented here have little to offer.

However for nonadjoint groups, although Theorems $B^{\prime}, C^{\prime}$ and $D^{\prime}$ do not apply directly, they may provide useful information; e.g. in [7] the author has calculated $J(x)$ for $S L(n, q)$ as a generalized Gaussian sum, using the analogue of Theorem $\mathrm{C}$ for $G L(n, q)$. The calculation is possible because in the above case one can prove:

* The restriction of $J$ to $U$ contains precisely one T-orbit of regular linear characters of $U$, and no other linear characters.

It is an open question whether * holds in general, i.e. for all (possibly nonadjoint) groups $\mathbf{G}$.

ADDED IN PROOF. Some of the results have been independently obtained

by N. Kawanaka in Unipotent elements and characters of finite Chevalley groups, Osaka J. Math. (to appear).

\section{REFERENCES}

1. A. Borel and T. A. Springer, Rationality properties of linear algebraic groups. II, Tôhoku Math. J. (2) 20 (1968), 443-497. MR 39 \#5576.

2. A. Borel and J. Tits, Groupes réductifs, Inst. Hautes Etudes Sci. Publ. Math. No. 27 (1965), 55-150. MR 34 \#7527.

3. R. Carter, Simple groups of Lie type, Wiley, New York, 1972.

4. C. Chevalley, Séminaire sur la classification des groupes de Lie algébriques, 2 vols., Secrétariat mathématique, Paris, 1958. MR 21 \#5696.

5. I. M. Gel'fand and M. I. Graev, Construction of irreducible representations of simple algebraic groups over a finite field, Dokl. Akad. Nauk SSSR 147 (1962), 529-532 = Soviet Math. Dokl. 3 (1962), 1646-1648. MR 26 \#6271.

6. R. B. Howlett, On the degrees of Steinberg characters of Chevalley groups, Math. Z. 135 (1974), 125-135.

7. G. I. Lehrer, Discrete series and regular unipotent elements, J. London Math. Soc. (2) 6 (1973), 732-736. MR 47 \#6835.

8. T. A. Springer, Some arithmetical results on semi-simple Lie algebras, Inst. Hautes Etudes Sci. Publ. Math. No. 30 (1966), 115-141. MR 34 \#5993.

9. T. A. Springer and R. Steinberg, Conjugacy classes, Seminar on Algebraic Groups and Related Finite Groups (Inst. for Adv. Study, Princeton, N. J., 1968/69), Lecture Notes in Math., vol. 131, Springer-Verlag, Berlin and New York, 1970, pp. 167-266. MR 42 \#3091.

10. R. Steinberg, Endomorphisms of linear algebraic groups, Mem. Amer. Math. Soc. No. 80 (1968). MR 37 \#6288.

11. Regular elements of semisimple algebraic groups, Inst. Hautes Études Sci. Publ. Math. No. 25 (1965), 49-80. MR 31 \#4788.

12. - Lectures on Chevalley groups, Mimeographed notes, Yale University, 1967.

DEPARTMENT OF PURE MATHEMATICS, UNIVERSITY OF SYDNEY, SYDNEY, N.S.W. 2006, AUSTRALIA 